Disclosure: No significant relationships.

Keywords: PSYCHOLOGICAL WELL-BEING; comorbidity; Internet Gaming Disorder; Vulnerability to Stress

\section{Suicidology and suicide prevention}

\section{$\mathrm{O} 292$}

Suicide and self-harm in women with mental disorders during pregnancy and the year after birth

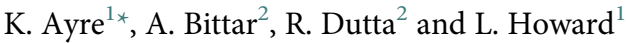 \\ ${ }^{1}$ Health Service And Population Research; Institute Of Psychiatry, \\ Psychology And Neuroscience, King's College London, SE AF, United \\ Kingdom and ${ }^{2}$ Institute Of Psychiatry, Psychology And Neuroscience, \\ Academic Department of Psychological Medicine, RJ, United Kingdom \\ ${ }^{\star}$ Corresponding author.
}

doi: 10.1192/j.eurpsy.2021.459

Introduction: There is little prospective data on the risk factors for later suicide in women who experience perinatal mental disorders, particularly beyond one-year postnatal.

Objectives: Among a cohort of women who were in contact with a mental healthcare provider during the perinatal period, to:

(1) Describe sociodemographic and clinical characteristics of the women who died by suicide (2) Understand when, in relation to childbirth, most suicides tended to occur.

Methods: Data-linkage of de-identified service-user electronic healthcare records, national hospital episode statistics and mortality data generated a cohort of women in contact with a mental healthcare service provider in London, UK, perinatally. Using Natural Language Processing and structured field extraction, we identified clinical, socio-demographic characteristics, self-harm exposure, and suicide.

Results: Among 5204 women, clinical and demographic characteristics of women who did and did not die by suicide were similar apart from indicators of illness severity including perinatal sedative medication prescription, clinician-rated functional impairment and smoking, which were more common in women who died by suicide. Suicide deaths occurred most frequently in the second year post-delivery. The most common method of suicide ocurring wihtin two years was by violent means, whereas after two years postnatal, the most common method was non-violent.

Conclusions: Our findings support the extension of perinatal mental healthcare service provision to two years post-delivery.

Disclosure: No significant relationships.

Keywords: Suicide; self-harm; perinatal; women's mental health

0293

Life stressors and resources as predictors of adolescent suicide attempt

E. Du Plessis

Psychology, University of the Free State, Bloemfontein, South Africa doi: 10.1192/j.eurpsy.2021.460

Introduction: Adolescent suicide poses a serious public health challenge. Several factors, such as early losses, discordant relationships, poverty, abuse and other life crises have previously been associated with the rise in adolescent suicides. However, a dearth of information exists regarding South African research on adolescent suicide.

Objectives: This study investigated the role of gender, race and psychosocial stressors and resources in attempted suicide among 1033 South African adolescents.

Methods: Using a cross-sectional research design, participants completed a biographical questionnaire and the Life Stressors and Social Resources Inventory, Youth Form. Logistic regression analysis was used to identify which stressors, resources and demographic variables, best predicted attempted suicide among the sample.

Results: The findings suggest that $12.5 \%$ (129) of the sample had previously attempted suicide. Being of mixed race $(\mathrm{p} \leq .01)$ and female $(\mathrm{p} \leq .01)$ significantly increased the likelihood of attempting suicide. Stressors associated with the increased likelihood of attempting suicide were Parents $(\mathrm{p} \leq .05)$, Extended Family $(\mathrm{p} \leq$ $.01)$, Home and Money ( $\mathrm{p} \leq .05)$, and Negative Life Events ( $\mathrm{p} \leq .01)$. Resources associated with the reduced likelihood of attempting suicide were supportive relationships with Parents $(\mathrm{p} \leq .01)$, with Boyfriend/Girlfriend $(\mathrm{p} \leq .01)$ and Positive Life Events $(\mathrm{p} \leq .01)$. Conclusions: These findings highlight the importance of supportive relationships and stable home conditions for the well being of adolescents.

Disclosure: No significant relationships.

Keywords: life stressors; adolescence; Suicide; ethnic

\section{4}

Trends in suicides among italian youth aged 10 to 25: A nationwide register study

A. Forte ${ }^{1,2 *}$, M. Vichi ${ }^{3}$, S. Ghirini ${ }^{3}$, M. Orri $^{4}$ and M. Pompili ${ }^{5}$

${ }^{1}$ Suicide Prevention Center, Sapienza University of Rome, Italy, Rome, Italy; ${ }^{2}$ Department Of Neurosciences, Mental Health And Sensory Organs, Suicide Prevention Center, Sant'andrea Hospital, Sapienza

University Of Rome, Rome, Italy, Sapienza university of Rome, ROMA, Italy; ${ }^{3}$ Statistical Service, Istituto Superiore di Sanità, National Institute of Health (ISS), Rome, Italy; ${ }^{4}$ Department Of Psychiatry, McGill Group for Suicide Studies, Douglas Mental Health University Institute, McGill University, Montreal, Canada, Montreal, Canada and ${ }^{5}$ Department Of Neurosciences, Mental Health And Sensory Organs, Suicide

Prevention Center, Sant'andrea Hospital, Sapienza University Of

Rome, Rome, Italy, Sapienza university of Rome, roma, Italy

${ }^{\star}$ Corresponding author.

doi: 10.1192/j.eurpsy.2021.461

Introduction: Suicide continues to be a significant cause of mortality in most countries worldwide, especially among youth. Documenting current trends and sources of variation in youth suicide rates is critical to inform prevention strategies.

Objectives: We aimed to 1 . document suicide mortality trends among Italian youth from 1981 to 2016 2. describe age, sex, and urbanization specific suicide rates in this period, and 3 . describe suicide methods and their change over time.

Methods: We relyed on official mortality data for the period 19812016 for adolescents and young adults (ages 10-25 years). We estimated standardized all-cause and suicide mortality rates per 100,000 individuals and used Joinpoint regression analysis to determine annual mortality trends and statistically significant changes in rate trends. Analyses were reported by sex, age group, urbanization level and suicide method. 